\title{
Caída del paciente adulto mayor hospitalizado
}

\author{
Fall of the hospitalized elderly patient
}

Quedas no paciente idoso hospitalizado

\author{
Alicia Fuentes Méndez \\ fuentesmendezalicia@gmail.com \\ https://orcid.org/0000-0003-2031-0420
}

Caja Nacional de Salud, Potosí, Bolivia

Recibido 4 de octubre 2021 / Arbitrado y aceptado 12 de noviembre 2021 / Publicado 30 de diciembre 2021

\begin{abstract}
RESUMEN
Las caídas en pacientes adultos mayores durante su estadía hospitalaria, son un hecho real que constituye un problema de salud, por su repercusión tanto a nivel personal como en costos institucionales, siendo la convergencia de distintos factores que las favorecen. El reconocer una caída es fundamental para poder instaurar oportunamente medidas destinadas a prevenirlas, así como para disminuir el daño secundario que pudiera alterar la rehabilitación del paciente. Objetivo. Proponer un protocolo de atención de enfermería para contribuir a prevenir la caída del adulto mayor durante su estadía hospitalaria. Materiales y Métodos: Se aplicaron las técnicas de la encuesta, entrevista y revisión de historiales clínicos, con la finalidad de conocer factores de riesgo que causan la caída y la percepsión sobre la atención resivida por el profesional al paciente durante su estadía hospitalaria. Resultados. La revisión de historiales clínicos evidenció el $10 \%$ de caídas, la encuesta aplicada reveló el $40 \%$ de factores extrínsecos, el $60 \%$ factores intrinsecos presentes y latentes en el hospital que ocacionan la caída, a la entrevista a pacientes adultos mayores manifiestan que por la sobrecarga de actividades y demanda de pacientes el profesional de enfermería no coadyuva con las necesidades de salud que ellos requieren, se sientes solos y abandonados. Conclusiones: Los resultados obtenidos de la investigación, fueron el camino para plantear una propuesta, sustentada en un modelo teórico que fundamenta e idealiza al proceso de atención de enfermería con sus componentes, para luego concretizar en un protocolo como documento normativo sustentado en la premisa del cuidado con calidad.
\end{abstract}

Palabras clave: Adulto mayor; caída intrahospitalaria; protocolo de prevención; seguridad del paciente

\begin{abstract}
Falls in elderly patients during their hospital stay are a real fact that constitutes a health problem, due to their repercussion both at a personal level and in institutional costs, being the convergence of different factors that favor them. Recognizing a fall is fundamental to be able to establish timely measures aimed at preventing them, as well as to reduce the secondary damage that could alter the patient's rehabilitation. Objective. To propose a nursing care protocol to help prevent falls in the elderly during their hospital stay. Materials and Methods. Survey, interview and clinical history review techniques were applied in order to determine the risk factors that cause falls and the perception of the care received by the professional during the patient's hospital stay. Results. The review of clinical histories evidenced $10 \%$ of falls, the survey applied revealed $40 \%$ of extrinsic factors, $60 \%$ intrinsic factors present and latent in the hospital that cause the fall, to the interview to elderly patients manifest that due to the overload of activities and demand of patients the nursing professional does not assist with the health needs they require, they feel alone and abandoned. Conclusions. The results obtained from the research, were the way to raise a proposal, based on a theoretical model that supports and idealizes the nursing care process with its components, and then materialize in a protocol as a normative document based on the premise of quality care.
\end{abstract}

Key words: Elderly; in-hospital fall; prevention protocol; patient safety 


\section{RESUMO}

As quedas em pacientes adultos mais velhos durante sua internação hospitalar são um fato real que constitui um problema de saúde, devido a suas repercussões tanto em nível pessoal quanto em termos de custos institucionais, sendo a convergência de diferentes fatores que os favorecem. Reconhecer uma queda é fundamental para poder implementar medidas destinadas a preveni-la em tempo hábil, assim como para reduzir os danos secundários que poderiam alterar a reabilitação do paciente. Objetivo. Propor um protocolo de cuidados de enfermagem para ajudar a prevenir quedas em idosos durante sua estadia hospitalar. Materiais e Métodos. Foram aplicadas técnicas de pesquisa, entrevista e revisão do histórico clínico para determinar os fatores de risco que causam as quedas e a percepção dos cuidados recebidos pelo profissional durante a permanência do paciente no hospital. Resultados. A revisão das histórias clínicas evidenciou $10 \%$ das quedas, a pesquisa aplicada revelou $40 \%$ de fatores extrínsecos, $60 \%$ de fatores intrínsecos presentes e latentes no hospital que causam a queda, na entrevista com pacientes adultos mais velhos eles afirmam que devido à sobrecarga de atividades e demanda dos pacientes o profissional de enfermagem não ajuda nas necessidades de saúde que eles necessitam, eles se sentem sozinhos e abandonados. Conclusões. Os resultados obtidos com a pesquisa, foram a forma de propor uma proposta, baseada em um modelo teórico que apóia e idealiza o processo de cuidados de enfermagem com seus componentes, para então se materializar em um protocolo como um documento normativo baseado na premissa de cuidados de qualidade.

Palavras-chave: Adulto idoso; queda hospitalar; protocolo de prevenção; segurança do paciente

\section{INTRODUCCIÓN}

En los últimos cien años la ancianidad se ha convertido en un problema social importante. Los adultos mayores son especialmente sensibles a disminuir su capacidad locomotora, iniciando de esta forma un progresivo deterioro del estado de funcionalidad física, psíquica y social (1).

La OMS define a la "caída como un acontecimiento involuntario, que hace perder el equilibrio y dar con el cuerpo en tierra" (2). Por tanto, todos los pacientes que ingresan a ser atendidos en los servicios de hospitalización, presentan riesgo potencial de sufrir una caída, por lo cual es necesario que el profesional de enfermería aprenda a reconocer los factores que los propician e identificar a los pacientes con mayor riego de sufrir un evento adverso (3). Al evitar las caídas se eliminan sus consecuencias, mejorando la calidad asistencial. El profesional de enfermería, como líder del equipo de salud, debe realizar una valoración al paciente hospitalizado e identificar el riesgo de sufrir una caída, para disminuir la morbilidad y los costos hospitalarios que esto implica (4).

Lo que indica; las caídas generan un daño adicional para el paciente, pudiendo ocasionar lesiones serias, incapacidad y en algunos casos la muerte. Tienen diferentes repercusiones como: aspectos físicos (complicaciones en su estado de salud, fracturas, daño en tejidos blandos, etc.), psicológicos (temor y ansiedad antelascaídas), sociales(familia excesivamente protectora); también repercuten en los costos de atención de las instituciones prestadoras y aseguradoras. Son una causa importante de discapacidad en las personas mayores, y a su vez uno de los desenlaces adversos de la fragilidad (4).

Son acontecimientos involuntarios que hacen perder el equilibrio y dar con el cuerpo en tierra u otra superficie firme que lo detenga, vistas además como un gran problema de salud que ha ido cobrando protagonismo en los últimos años, sobre todo, al actual envejecimiento de la población. 
Por ello es importante reconocer el riesgo de caídas en un paciente es fundamental, para poder instaurar oportunamente medidas destinadas a prevenirlas, así como para disminuir el riesgo de daño secundario, que pudiese alterar la rehabilitación y producir la morbilidad del paciente (5).

Por lo tanto, la participación de enfermería, es "fundamental en la promoción de salud del adulto mayor, enfocado en la prevención de las caídas" (6).

El profesional al comunicarse e interactuar con las personas de edad avanzada y su familia, en diferentes ámbitos laborales como: hospitales, asilos, centros de día, puede valorar e identificar los riesgos de la población e influir en la promoción de estilos de vida saludables (7).

En el contexto hospitalario, el foco de atención es el cuidado al paciente hospitalizado y sus necesidades, lo que implica identificar constantemente los elementos, que influyen en la salud del paciente (8). Donde el profesional de enfermería, es eje principal en la prevención de caídas, por ser una profesión que dirige sus acciones al individuo de forma integral, proporcionando un cuidado seguro a los pacientes que ingresan a ser atendidos en los nosocomios (9) es necesario que enfermería, identifique los eventos adversos, entendidos como aquella lesión no intencionada que se presenta en el proceso asistencial.

Se ha ejercitado en el último medio siglo un avance notable, existen múltiples ocasiones, en las que ante un mismo problema se actúa de forma diferente (10), enfermería no es ajena a estos avances en la ciencia siendo una profesión de ayuda con conocimientos abstractos formado por un aserie de teorías y modelos enriquecidos que se obtienen de la investigación (11).

En este sentido, se ha visto la necesidad de contar con protocolos de atención de enfermería, como documentos normativos que ayuden al profesional de enfermería, a tomar conductas de manera normada y consensuada, en el cuidado al paciente adulto mayor, brindando de esta manera una seguridad intrahospitalaria.

\section{MATERIALES Y MÉTODOS}

La metodología aplicada en el estudio se trató de una investigación documental bajo el análisis mixto de tipo descriptivo, transversal, retrospectiva, a través del método empírico, con la aplicación de las técnicas de la encuesta, entrevista y revisión de historiales clínicos del primer semestre de la gestión 2020. Que revelaron la situación actual de la atención de enfermería al adulto mayor durante su estadía hospitalaria. La sobrecarga de actividades, demanda de pacientes, hace que enfermería no logre valorar todo aspecto físico y psicológico del paciente, desde el momento de su hospitalización, no realice diagnósticos reales para poner en ejecución cada una de las acciones a favor del paciente, menos aún evalué, siendo este un riesgo potencial para el cuidado de los pacientes, además de los factores intrinsecos y extrínsecos que afectan la calidad de atención. 
El universo fue conformado por 267 pacientes hospitalizados, también se tomó una muestra censal de 6 profesionales que representan el $100 \%$ de enfermería. La muestra es seleccionada a través de la técnica no probabilistica por conveniencia en pacientes adultos mayores hospitalizados.

Criterios de exclusión. Donde se generalizó a toda la población adulto mayor que pudiera brindar información capaz de dialogar. Se seleccionó al profesional de enfermería que cumple funciones en los diferentes turnos del servicio de "Medicina Interna".

Criterios de inclusión. Se determinó la participación del profesional de enfermería quienes son los que ofrecen atención de salud directo a pacientes hospitalizados. Asimismo, a pacientes adultos mayores hospitalizados en el lapso del primer semestre de la gestión 2020 que puedan entablar conversación sin ningún problema.

\section{RESULTADOS}

\section{Revisión de historiales clínicos de pacientes adultos mayores hospitalizados en el servicio de "Medicina Interna"}

Se revisaron 122 historiales clínicos de pacientes adultos mayores hospitalizados del primer semestre de la gestión 2020; con la finalidad de conocer: Número de pacientes hospitalizados, diagnósticos con los que ingresaron y las complicaciones que sufrieron por una caída los adultos mayores durante su estadía hospitalaria.

En la Tabla 1 se muestra el número de pacientes internados por edad y sexo. Donde se evidencia, que en el sexo femenino existe un índice mayor de afección, esto por la fragilidad que presenta, aunque estos resultados son contradictorios, tienden a señalar una incidencia equivalente en ambos sexos o en otros casos superior en los varones.

Tabla 1. Número de pacientes internados por edad y sexo en el servicio de "Medicina Interna". Datos recados de historiales clínicos.

\begin{tabular}{ccccccc}
\hline \multirow{2}{*}{ EDAD } & MASCULINO & $\%$ & FEMENINO & $\%$ & TOTAL & $\%$ \\
\hline $60-64$ & 10 & 8 & 14 & 12 & 24 & 20 \\
$65-69$ & 12 & 10 & 12 & 10 & 24 & 20 \\
$70-74$ & 1 & 1 & 10 & 8 & 11 & 9 \\
$75-79$ & 10 & 8 & 15 & 12 & 25 & 20 \\
$80-84$ & 4 & 3 & 17 & 14 & 21 & 17 \\
$85-89$ & 3 & 2 & 8 & 7 & 11 & 9 \\
$90-$ más & 2 & 2 & 4 & 3 & 6 & 5 \\
\hline TOTAL & $\mathbf{4 2}$ & $\mathbf{3 4}$ & $\mathbf{8 0}$ & $\mathbf{6 6}$ & $\mathbf{1 2 2}$ & $\mathbf{1 0 0}$ \\
\hline
\end{tabular}


En la Tabla 2 se muestra los diagnósticos con los que ingresaron los pacientes al servicio de "Medicina Interna". El adulto mayor es más susceptible de sufrir diferentes enfermedades, como problemas de osteoporosis, diabetes, problemas del corazón, neumonía, desequilibrios alimentarios, otras patologías como la incontinencia vesical, artrosis $\mathrm{y} / \mathrm{o}$ artritis, enfermedades mentales, patologías muy frecuentes en esta edad, que son factores preponderantes a ocasionar una caída, trayendo como consecuencias lesiones físicas y psicológicas.

Tabla 2. Diagnósticos con los que ingresan a ser hospitalizados los pacientes adultos Mayores. Datos recabados en historiales clínicos.

\begin{tabular}{lcc}
\hline \multicolumn{1}{c}{ DIAGNÓSTICOS } & CANTIDAD & PORCENTAJE \\
\hline Artritis y artrosis & 3 & 2 \\
Enfermedades mentales & 2 & 1 \\
Osteoporosis & 30 & 24 \\
Diabetes & 23 & 19 \\
Desequilibrios alimentarios & 12 & 10 \\
Neumonías & 20 & 17 \\
Problemas del corazón & 22 & 18 \\
Otro (incontinencia vesical) & 10 & 9 \\
\hline TOTAL & $\mathbf{1 2 2}$ & $\mathbf{1 0 0}$ \\
\hline
\end{tabular}

La Tabla 3 evidencia que los pacientes adultos mayores se caen más a menudo mientras: deambulan, al realizar cambios de posición, pararse, sentarse y en gran parte sucede en la ausencia del personal de salud o en una actividad no asistida como: trasladarse desde o hacia la cama, camilla o sillas de ruedas. Lo que demuestra la falta de una atención de enfermería orientada por protocolos estandarizados, que puedan velar la integridad física y psicológica del paciente durante su estadía hospitalaria.

Tabla 3. Caídas que sufrieron los adultos mayores durante su estadía hospitalaria. Datos recabados de historiales clínicos.

\begin{tabular}{ccc}
\hline COMPLICACIONES & CANTIDAD & PORCENTAJE \\
\hline SÍ & 12 & 10 \\
NINGUNA & 110 & 90 \\
\hline TOTAL & $\mathbf{1 2 2}$ & $\mathbf{1 0 0}$ \\
\hline
\end{tabular}


Resultados de la encuesta aplicada a profesionales de enfermería que cumplen funciones al cuidado del paciente

La encuesta se aplicó a 6 profesionales de enfermería, para conocer la situación actual del proceso de atención de enfermería (PAE) al adulto mayor, a partir de las etapas que se contemplan: valoración, diagnóstico, planificación, ejecución, evaluación y teniendo en cuenta los factores de riesgo intrínsecos y extrínsecos, causantes de sufrir un evento adverso como la caída en el paciente adulto mayor durante su estadía hospitalaria.

El Gráfico 1 destaca el manejo del Proceso de Atención de Enfermería en el adulto mayor durante su estadía hospitalaria, lo cual revela, el $80 \%$ el profesional no lo realizan, por la sobrecarga de actividades y demanda de pacientes, el $20 \%$ sí lo aplican ya que es un método científico que ayuda al profesional a brindar cuidados asistenciales con calidad.

Gráfico 1. Manejo del proceso de atención de enfermería en el paciente adulto mayor. Datos recabados de la encuesta a profesionales de enfermería.

En el Gráfico 2 se muestran los factores intrínsecos (propios del paciente, como las enfermedades crónicas, la polifarmacia) y extrínsecos (relacionados al entorno del paciente, lo ambiental hospitalario), causantes de sufrir una caída en paciente adulto mayor hospitalizado trayendo consecuencias físicas (fracturas, lesiones, contusiones), psicológicas (síndrome post caída). 


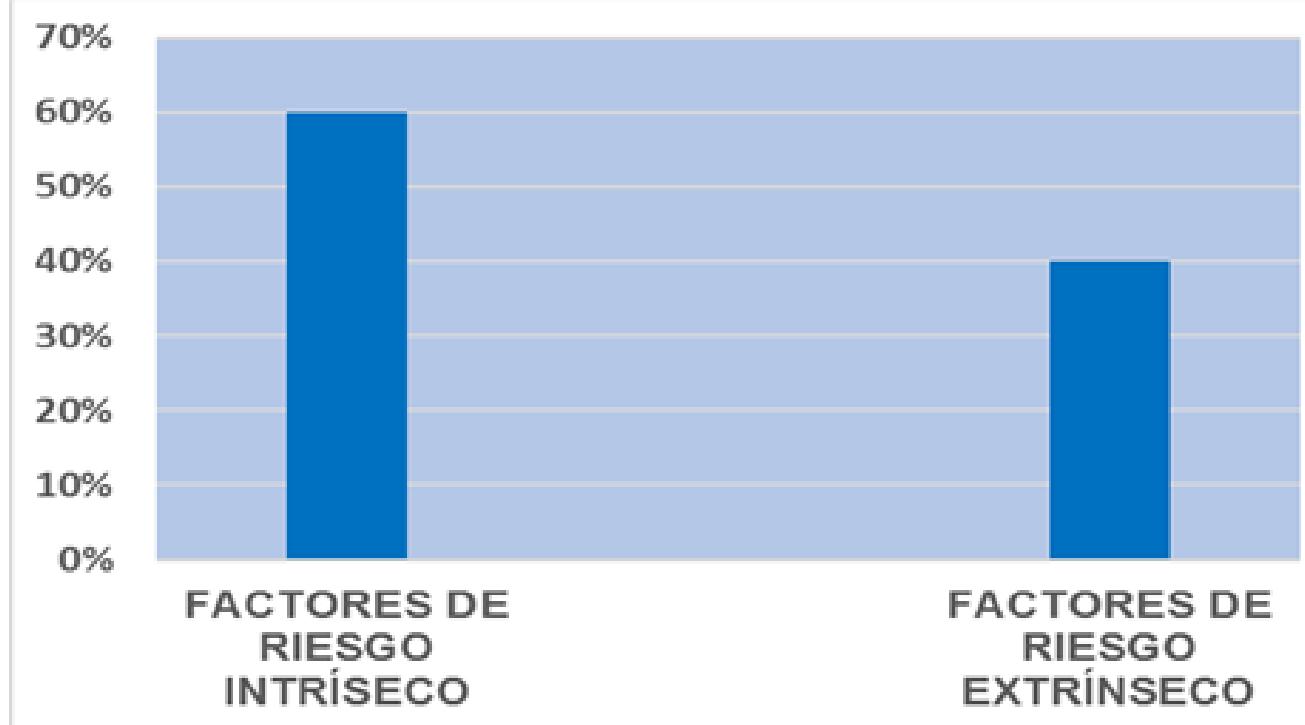

Gráfico 2. Factores de riesgo causantes de sufrir una caída en el paciente adulto mayor hospitalizado. Datos recabados de la encuesta aplicada al profesional de enfermería.

La Tabla 4, refiere a la interrogante sobre la existencia o no de un protocolo como documento normativo para la atención de enfermería. El 100\% del profesional expreso que no se tiene este documento, lo cual dificulta poder reglar y normar los procedimientos a realizar en el paciente adulto mayor hospitalizado.

Tabla 4. Existencia de un protocolo como documento normativo para el cuidado del paciente adulto mayor hospitalizado. Datos recabados de la encuesta aplicada al profesional de enfermería.

\begin{tabular}{cccc}
\hline EXISTENCIA DE UN PROTOCOLO DE ATENCIÓN DE ENFERMERÍA & CANTIDAD & PORCENTAJE \\
\hline SÍ & 0 & 0 \\
NO & 6 & 100 \\
\hline TOTAL & 6 & 100 \\
\hline
\end{tabular}

La Tabla 5 revela que el profesional de cuidados de enfermería teniendo como considera la necesidad de contar con un base el conocimiento científico, así como protocolo de atención como documento también dar y mejorar la seguridad del usuario normativo para la práctica, instrumento que constituye una importante fuente de información donde permite unificar criterios y del personal de salud. También forma parte de protección a futuras represalias legales como la omisión o negligencia. 
Tabla 5. Consideración de la existencia de un protocolo de atención de enfermería para prevenir la caída del paciente adulto mayor hospitalizado.

\begin{tabular}{ccc}
\hline NECESIDAD DE LA EXISTENCIA DE UN PROTOCOLO DE ATENCIÓN & CANTIDAD & PORCENTAJE \\
\hline Sí & 1 & 17 \\
NO & 5 & 83 \\
\hline TOTAL & 6 & 100 \\
\hline
\end{tabular}

Resultados de la entrevista aplicada a pacientes adultos mayores hospitalizados

La entrevista se aplicó a 122 pacientes hospitalizados en el primer semestre de la gestión 2020, tomando como muestra la técnica no probabilística por conveniencia de adultos mayores, con la finalidad de conocer la percepción que tienen sobre la atención recibida por el profesional de enfermería y la información sobre los factores de riesgo que causan un evento adverso como la caída.

Al recoger la información ofrecida se conoce que en el $90 \%$ considera que, en el momento de recibir la atención de salud, enfermería no toma en cuenta la edad que presenta el paciente, con el paso de los años incrementan los niveles de discapacidad de los adultos mayores. El $10 \%$ restante expresa que la atención de salud por el profesional de enfermería es oportuno, recibiendo los cuidados necesarios para mitigar su dolencia. $\mathrm{El}$ $60 \%$ considera que el profesional de enfermería al momento de la admisión del adulto mayor no tiene en cuenta la enfermedad y la dolencia que padece el paciente, hasta en el momento de su valoración con el médico. El 40\% restante manifestó recibir respeto, solidaridad, empatía por parte del profesional de enfermería en el momento de su internación. El 50\% considera que la infraestructura hospitalaria es adecuada cuenta con todos los servicios básicos. El 50 \% restante expreso que los ambientes son fríos, hostiles, existiendo llanto y dolor, la iluminación de los focos son bajos, no permiten ver con claridad. Ítem 5: El 70\% señala que no recibe información al momento de su internación, como: las condiciones de sus camas, funcionamiento de timbres. El 30\% restante expreso que enfermería, responde sobre algún interrogante como: la ubicación o funcionamiento de algún objeto. El $90 \%$ manifestó al momento de su hospitalización el personal le otorga la ropa de cama y otros enseres, acostumbrándose a su uso. El 10\% restante expreso tener su propia ropa de cama, siendo cómoda y acorde a la altura y talla que tiene cada persona.

\section{Discusión}

Un número significativo de pacientes adultos mayores que asisten a un nosocomio, tienen riesgo de sufrir una caída en cualquier momento de su hospitalización. El proceso de envejecimiento se asocia con varios cambios que predisponen a las caídas (2). 
El mayor índice de caídas que revela el estudio de investigación comprende entre las edades de 75-79 años. Las patologías más prevalentes en los ancianos que sufren las caídas son la osteoporosis seguido de la diabetes, problemas del corazón y otros, a la revisión de historiales clínicos evidencia la caída del paciente durante su estadía hospitalaria en el servicio de "Medicina Interna de la Caja Nacional de Salud".

El adulto mayor que presenta caídas tienen un promedio 3 a 4 enfermedades coexistentes, como las causas cardiovasculares (generalmente provocan síncopes o mareos), neurológicas (epilepsia, alteraciones cognitivas, cuadros confusionales, alzhéimer), osteoarticulares (osteoporosis, alteraciones de los pies), polifarmacia (ingesta de 4 o más medicamentos) (4).

A mayor edad, más riesgo de caídas. Los adultos mayores se caen más a menudo cuando: deambulan, al realizar cambios de posición, pararse, sentarse y en gran parte sucede en la ausencia del personal de salud o en una actividad no asistida como: trasladarse desde o hacia la cama, camilla o sillas de ruedas. Lo que demuestra la falta de una atención por enfermería orientada por protocolos estandarizados, que puedan velar la integridad física y psicológica de los adultos mayores durante su estadía hospitalaria.

El $80 \%$ del profesional de enfermería por la sobrecarga de actividades o demanda de pacientes no logra realizar una valoración exhaustiva de todo aspecto físico y psicológico del paciente adulto mayor desde el momento de su admición y hospitalación lo cual hace que no se pueda plasmar un diagnóstico de enfermería, la valoración lo realiza de forma superficial o no lo realizan por lo tanto los diagnósticos planteados no son correctos o guían hacia un plan correcto, el profesional no cuenta con planes de cuidados que estén estandarizados y protocolizados, por lo tanto los procedimientos de enfermería no se ejecutan tal como son. no realiza una evaluación continua del efecto de las acciones aplicadas en el cuidado por enfermería. Siendo una acción de enfermería indispensable para poder ajustar las acciones a favor del paciente adulto mayor hospitalizado.

Las causas de sufrir una caída en el paciente adulto mayor durante su estadía hospitalaria, son multifactoriales entre ellas los factores de riesgo intrinsecos en el $60 \%$ y extrínsecos en el $40 \%$ que se encuentran presentes latentes en el servicio de "Medicina Interna" para ocasionar el evento adverso.

El adulto mayor es un ser humano que integra un grupo de riesgo para múltiples enfermedades y disfunciones orgánicas, derivadas del proceso natural de envejecimiento. Este hecho, hace del adulto mayor una persona frágil, cuya integridad física, psicológica, social y espiritual, se ve en constante riesgo de ser afectada por factores externos (5).

Si bien la etiología de la caída en el adulto mayor es multifactorial, se han identificado factores de riesgo condicionantes ya sea externos o modificables, de tipo 
medioambiental, factores internos o no modificables como el propio envejecimiento - la existencia de enfermedades crónicas o agudas (3). Las caídas ocacionan lesiones leves como hematomas, lesiones graves como fracturas de cadera, que son mayor incidencia en pacientes que sufren este evento adverso (4).

El riesgo de caídas es un problema frecuente en las personas mayores. La realización de una valoración sistemática de este riesgo, a través del manejo de un instrumento específico como el protocolo de atención de enfermería, pueda contribuir a prevenir la caída del paciente identificando precozmente el nivel de riesgo y sus factores que ocacionan este evento adverso.

Los protocolos de enfermería son documentos que además de establecer una normativa para la práctica, constituye una importante fuente de información, instrumentos que permiten unificar criterios de cuidados de enfermería, teniendo como base el conocimiento científico, mejorando la seguridad del paciente y del personal de salud (10).

Por lo señalado, el paciente adulto mayor a la entrevista aplicada, expreso no estar seguro durante su estadía hospitalaria, se siente solo y abandonado porque el profesional de enfermería no coadyuva con las necesidades de salud que ellos requieren. Además de los factores de riesgo intrinsecos y extrínsecos presentes latentes en el centro hospitalario para que el paciente sufra una caída.

Cuidar a un paciente con una alteración de su estado de salud durante la aplicación de un tratamiento, que involucre su integridad física y psicológica, implica una serie de intervenciones que requieren una previa valoración clínica y la planeación de las acciones de cuidado para la implementación por parte del personal de enfermería (9).

La esencia del profesional es el cuidado al paciente, la función de enfermería, como cuidadora, implica la participación activa en la promoción, mantenimiento y recuperación de la salud, mediante medidas preventivas para evitar la aparición de la enfermedad, su progresión o prevenir secuelas asegurando la continuidad del cuidado, facilitando la independencia del individuo (9).

\section{CONCLUSIONES}

La caracterización de la situación actual del proceso de atención de enfermería, permitió verificar el problema existente, la caída del paciente adulto mayor durante su estadía hospitalaria, el profesional de enfermería no logra valorar todo aspecto físico, emocional y los factores de riesgo (intrínsecos y extrínsecos) causantes de sufrir un evento adverso. La caída se asocia con importantes consecuencias físicas y psicológicas. La participación de enfermería es fundamental en el cuidado, es la esencia de la profesión. Por tal motivo surge la necesidad de reflexionar acerca de la importancia del cuidado al paciente. El manejo del proceso de atención de enfermería con las cinco etapas (valoración, diagnóstico, planificación, ejecución y evaluación), permite al profesional realizar planes de cuidados de forma directa e individualizada, identificando las respuestas humanas que responden a estilos de vida que determinan la salud o enfermedad, de esta 
manera garantizar la calidad del cuidado, brindando una seguridad al paciente adulto mayor durante su estadía hospitalaria.

\section{Recomendaciones}

Realizar otros estudios sobre las caídas orientado a diferentes vertientes de la atención al paciente adulto mayor hospitalizado, sobre la necesidad de que enfermería sea un buen comunicador oral, incentivar el cambio de actitud y pensamiento del profesional para contribuir a garantizar la calidad del cuidado del individuo, familia y comunidad.

\section{REFERENCIAS BIBLIOGRÁFICAS}

1. Sandoval L, Capuñay J, Varela L. Caídas en el adulto mayor. Estudio de una serie de pacientes de consultorio externo de medicina del Hospital Nacional Cayetano Heredia: Rev. Med Herd. 1996; (7):07-16

2. Organización Mundial de la Salud. Caídas del adulto mayor [Internet] 26 de abril 202. Disponible en http://www. Who.int/es/newsroom.

3. Gonzales de la Cruz P, Solís L. Factores de riesgo de caídas e índice de masa corporal en el adulto mayor hospitalizado. Rev. Cuid. [Internet] 2019 [consultado 12 /06/2021]. Disponible en http//dx.doi.org/10.15649/ cuidarte.v10 [Link]

4. Silva F, Partezani R. Causas y factores asociados a las caídas del adulto mayor. Scielo. 2019;(16): 3-10
5. Cotaquispe A, Arévalo J. Riesgo de caídas en el adulto mayor hospitalizado. Ciencia y arte de enfermería. 2017;(2): 09-17

6. Miranda K, Rodríguez $\mathrm{Y}$, Cajachagua $M$. Proceso de atención de enfermería como instrumento del cuidado, significado para estudiantes del último curso. Enferm. Univ. [revista en la internet]. 2019;(16): 3-11

7. Bello N, Fenton M. Proceso de atención de enfermería, necesidad de cambio. Rev. Cubana Enferm. 1988; (13): 11-27

8. Lemus $N$, Linares Lázaro, Linares L, Macías L, Morales R. Comportamiento de las caídas en adultos mayores ingresados en servicio de Geriatría. Rev Ciencias Médicas. 2019; (23): 5-14

9. Abades M. Los cuidados enfermeros en los centros geriátricos según de Watson. Gerokomos. 2007;(18):3-5

10. Atienza M. Diseño y evaluación de un protocolo clínico. Elsevier. Medicina Integral. [Internet].2000 [consultado 12/08/2021]; 35(9): Disponible en: http://www.elsevier.es/esrevista-medicina-inegral.

11. Reconde D, Peña $M$. Las regularidades teóricas de los protocolos de actuación de enfermería como resultado científico enfermero. ENE. 2019; (13): 3-13

Conflicto de intereses: Ninguno declarado por los autores.

Financiación: Ninguna declarada por los autores.

Agradecimiento: Ninguno manifestado por los autores

\section{ACERCA DEL AUTOR}

Alicia Fuentes Méndez. Licenciada en Enfermería, Universidad San Francisco Xavier de Chuquisaca. Magister Médico Quirúrgico, Universidad Autónoma Tomás Frías, Caja Nacional de Salud, Bolivia. 